\title{
Distance from Home to Research Center: A Barrier to In-Person Visits but Not Treatment Adherence in a Stroke Trial
}

\author{
Enrique C. Leira ${ }^{a, b}$ Catherine M. Viscolid ${ }^{d}$ Linnea A. Polgreen ${ }^{c}$ Mark Gorman $^{e}$ \\ Walter N. Kernan ${ }^{d}$ on behalf of the IRIS Trial Investigators \\ ${ }^{a}$ Colleges of Medicine, lowa City, IA, USA; b Public Health, lowa City, IA, USA; CPharmacy, University of lowa, lowa \\ City, IA, USA; ${ }^{d}$ Yale School of Medicine, New Haven, CT, USA; ${ }^{e}$ Maine Medical Center, Portland, ME, USA
}

\section{Keywords}

Clinical trial · Prevention · Adherence · Distance ·

Geography $\cdot$ Ischemic stroke $\cdot$ Secondary prevention

\begin{abstract}
Background and Purpose: Clinical trials often seek to enroll patients from both urban and rural areas to safeguard the generalizability of results. However, maintaining contact with patients who live away from a recruitment site, including rural areas, can be challenging. In this research we examine the effect of distance between patient and study centers on treatment adherence and retention. Methods: Secondary analysis of 2,466 participants in the Insulin Resistance Intervention after Stroke trial who were enrolled from research sites in the United States. Driving distance between the zipcodes of patient's reported place of residence and the study center was calculated. Outcome measures were loss to follow-up, completion of annual in-person visits, adherence to preventive therapy, and adherence to study drug in the first 3 years of participation. Logistic regression models were
\end{abstract}

(c) 2018 S. Karger AG, Basel

E-Mail karger@karger.com www.karger.com/ned used to adjust for confounders. Results: Distance from residence to research center was not associated with loss to follow-up, adherence to study drug, or adherence to preventive therapy ( $p>0.05$ for each). However, patients who lived farther from the research center ( $>120$ miles), compared to patients who lived closer ( $<60$ miles), were less likely to complete the second annual in-person visit (62 vs. $81 \%$; adjusted OR $0.48 ; 95 \% \mathrm{Cl} 0.31-0.75$ ) and third visit (53 vs. $75 \%$; adjusted OR 0.44; 95\% Cl 0.29-0.67). Conclusions: Distance between patient and study center was an independent predictor of missed in-person visits but not with adherence to study treatment or preventive care.

(c) 2018 S. Karger AG, Basel

\section{Introduction}

Clinical trials investigators often seek to enroll diverse populations to maximize the generalizability of their results and conform to commonly accepted ethical standards of scientific research. Commonly, however, there is 
tension between a researcher's desire to enroll a diverse population and his or her desire to enroll participants who are thought to be most likely to comply with the study protocol, who are the easiest to follow, and who will remain involved until the research is completed $[1,2]$. Patients who are older in age, economically disadvantaged, homeless, cognitively challenged, medically complex, or who speak a foreign language are often omitted because of this tension. Another group that is commonly omitted comprises patients who live long distances from the research center, which is typically located in a city. In the United States, this particular practice may lead to the omission of up to $20 \%$ of research candidates who live in a rural area [3-5].

Long distance between a home and an urban research center is an absolute impediment to participating in many acute stroke trials that have a short time window for enrolment. Air transportation [6] or enrolment in the field [7] can help, but these strategies are seldom used. In contrast, long distance is not an absolute impediment to enrolment in most secondary prevention trials that typically have longer time windows for enrolment. In both acute and non-acute trials, research participants who live a longer distance from the research center may encounter inconvenience and risk as they travel to follow-up appointments. A common assumption is that they will opt out of research participation, or will withdraw from the research or stop adhering to treatments over time.

The assumption that travel distance adversely affects trial participation, however, has not been adequately examined. In this research, we aim to estimate the independent effect of distance from a research center on different aspects of clinical trial participation, including trial retention, adherence to study drug and best medical practices, and follow-up visits.

\section{Material and Methods}

We conducted a secondary analysis of the Insulin Resistance Intervention after Stroke (IRIS) trial, an international multicenter, randomized, placebo-controlled study that tested the effectiveness of pioglitazone for prevention of stroke or myocardial infarction in patients without diabetes after a recent TIA or ischemic stroke. The methods and the results of the trial have been described elsewhere $[8,9]$.

We examined the association between distance from residence to local IRIS study center and risk for participant loss, adherence to study drug, adherence to preventive therapy, and completion of study visits. Distance was calculated with Google Maps (Mountain View, CA, USA) using the zip-codes of the participant's current residence and the IRIS study center. For con- sistency in geographical structure, only participants enrolled from US sites (excluding Puerto Rico) were included in this analysis. Patients were excluded if they were transferred to another site during the trial or if address information was not provided by the enrolment site to the Coordinating Center. For each participant, the distance from home residence to the research site was calculated at the time of randomization and at dates for annual visits in years 1,2 , and 3 using the most updated address in the IRIS database.

A patient was classified as lost if he or she dropped out (i.e., withdrew consent) or could not be located (i.e., missed more than 13 months of follow-up). A patient was classified as adhering to prevention treatment if he or she achieved 3 prevention goals: blood pressure $<140 / 90 \mathrm{~mm} \mathrm{Hg}$, low-density lipoprotein (LDL) cholesterol $<2.59 \mathrm{mmol} / \mathrm{L}$, and on anticoagulant or antiplatelet therapy. A patient was classified as adhering to the experimental therapy if he or she reported taking the study drug. All outcomes were evaluated at years 1,2 , and 3 of trial participation, except loss to follow-up, which was measured over the duration of the trial.

\section{Statistical Analysis}

We identified the following baseline patient features as potentially related to distance and adherence/retention outcomes: age, sex, living alone, race (white, black, other, unknown), Hispanic ethnicity, years of education, (high school, college), region of the country (northeast, midwest, south, west), impaired cognition (Modified Mini Mental Status Examination score <89), disability (modified Rankin Scale score $\geq 3$ ), leg edema on examination, current smoker, aerobic exercise, prevention goals not met, study drug assignment (pioglitazone vs. placebo), and baseline visit conducted at the participant's home.

Because associations between driving distance and outcomes may be sensitive to the distance categories used, we employed 2 strategies. First, in categorical analysis, distance was defined as $0-59,60-119$, and $>120$ miles because these categories roughly translate to the number of hours traveled [10]. Second, we examined driving distance as a continuous variable. The associations between patient features and baseline distance strata were tested using the Chi-square statistic for proportions, and Wilcoxon ranksum measure for continuous variables. Logistic regression analysis was performed with distance included as both a categorical and continuous feature. Results are presented unadjusted and after adjustment for features identified in bivariate analysis as significantly $(p<0.05)$ related to baseline distance strata. All statistical analyses were performed using SAS version 9.3 (SAS Institute Inc., Cary, NC, USA). Ethics approval was obtained from the local Institutional Review Boards, and written informed consent was obtained from all IRIS patients.

\section{Results}

Of 3,876 participants in the IRIS trial, 2,572 were enrolled from sites in the continental United States. A total of 106 US participants were excluded from the current study (58 participants who were transferred between sites during the trial and 48 participants missing 
Fig. 1. Distribution of distance to site at baseline $(n=2,466)$.

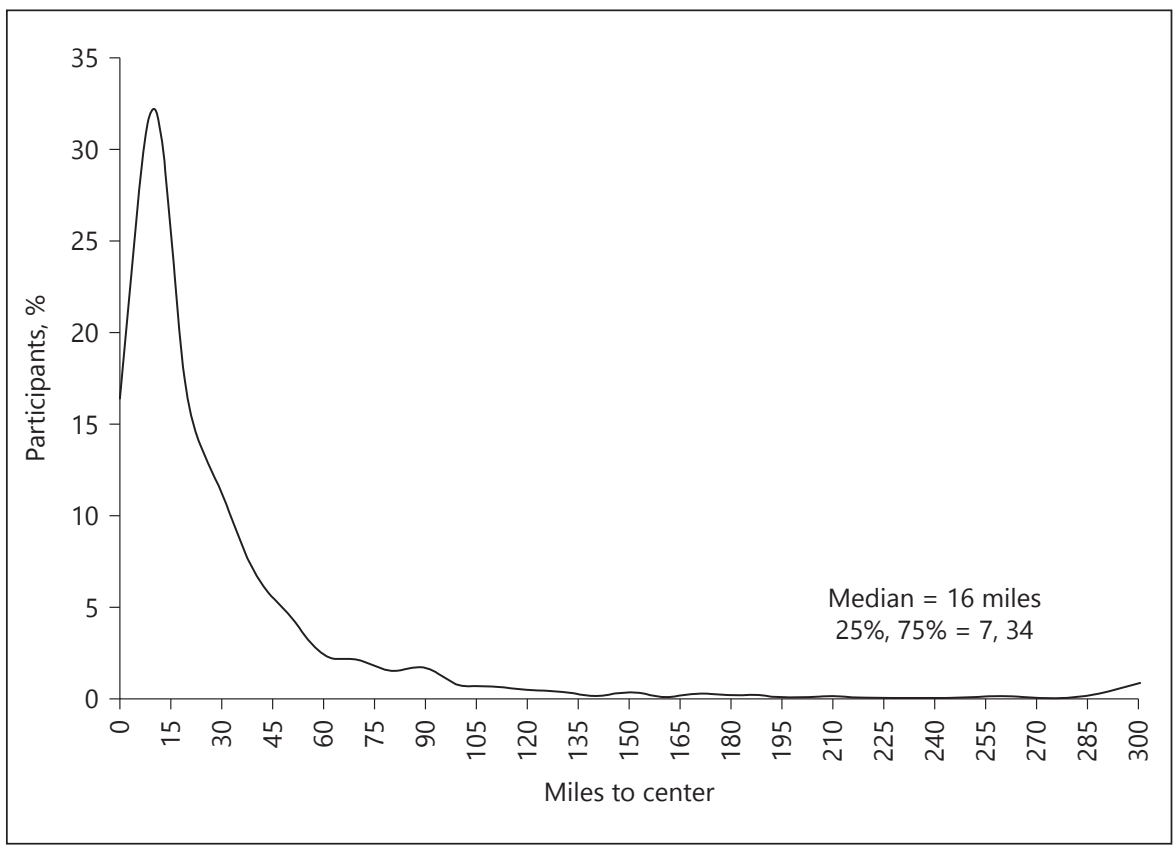

zip-code data). In IRIS, the median distance to site was 16 miles and only $11 \%$ of participants lived at least 60 miles from the research site when enrolled (Fig. 1). Table 1 shows the baseline characteristics of the study cohort stratified by driving distance from patient's home to the research site at the time of randomization. Compared to patients who lived closer, the 276 patients who lived $\geq 60$ miles distant were more likely to be white race, of the male gender, and likely to live in a rural area. They were less likely to live alone or be from the Northeast. The baseline modified Rankin Scale scores were similar in the 2 groups, indicating no "distance bias" that has been reported in other studies [11]. As expected, a greater proportion of more distant participants lived in a rural location, and sites located in the western states enrolled more distant participants compared to other regions.

The proportion of participants who became lost-tofollow-up was greater for patients who lived within 60 miles of the site (11\%), compared to patients who lived at greater distances (6\% for $60-119$ miles; $4 \%$ for $120+$ miles; chi-square test, $p=0.007)$. This difference remained statistically significant after adjustment for baseline features related to distance from site $(p=0.003$; Table 2). When distance was considered on a continuous scale, the effect was in the same direction, but the finding was attenuated (adjusted OR per 30 miles, 0.96; $p=0.06)$.
At year 1,89\% of participants within 60 miles of the site completed the annual visit in person, compared to 87 and $80 \%$ for participants living $60-120$ and $120+$ miles distant respectively $(p=0.03)$. The finding in year 1 was not significant after adjustment for baseline features. However, a reduced rate of visit completion was observed at years 2 and 3, both in the stratified and continuous analyses. In contrast, rates of risk factor control and being part of an ongoing study were not significantly associated with distance to site (Tables 2, 3).

\section{Discussion}

In the IRIS trial, $11 \%$ of US participants lived at a distance that took an hour or more driving time from their enrolment site at that time of randomization. These participants were less likely to return to the site for completion of required annual, in-person visits compared to participants who lived closer. This negative effect increased with the duration of follow-up, suggesting that the burden of driving was amplified over time [12]. However, participants who lived further away from the enrolment site were more likely to remain in the trial, and just as likely as more proximate participants to stay on study drug and meet secondary prevention goals.

Our findings suggest that trial patients who live at greater distances from an enrolment site may contribute 
Table 1. Baseline features of study cohort by driving distance strata

\begin{tabular}{|c|c|c|c|c|}
\hline \multirow[t]{2}{*}{ Baseline feature } & \multicolumn{3}{|c|}{ Distance from site at randomization } & \multirow[t]{2}{*}{$p$ value $^{*}$} \\
\hline & $\begin{array}{l}0-59 \text { miles } \\
(n=2,190)\end{array}$ & $\begin{array}{l}60-119 \text { miles } \\
(n=199)\end{array}$ & $\begin{array}{l}120+\text { miles } \\
(n=77)\end{array}$ & \\
\hline Age, years & $63(55-71)$ & $63(57-68)$ & $59(54-66)$ & 0.09 \\
\hline$<60$ & $885(40)$ & $70(35)$ & $40(52)$ & 0.004 \\
\hline $60-69$ & $691(32)$ & $83(42)$ & $26(34)$ & \\
\hline $70-79$ & $447(20)$ & $43(22)$ & $7(9)$ & \\
\hline $80+$ & $167(8)$ & $3(2)$ & $4(5)$ & \\
\hline Gender, male & $1,353(62)$ & $144(72)$ & $61(79)$ & 0.0002 \\
\hline Lived alone & $726(33)$ & $36(18)$ & $16(21)$ & $<0.0001$ \\
\hline Race & & & & $<0.0001$ \\
\hline White & $1,704(78)$ & $183(92)$ & $64(83)$ & \\
\hline Black & $376(17)$ & $7(4)$ & $8(10)$ & \\
\hline Other & $72(3)$ & $7(4)$ & $4(5)$ & \\
\hline Unknown & $38(2)$ & $2(1)$ & $1(1)$ & \\
\hline Hispanic ethnicity & $94(4)$ & $2(1)$ & $2(3)$ & 0.06 \\
\hline Education, years & $13(12-16)$ & $12(12-16)$ & $12.5(12-15)$ & 0.90 \\
\hline$<$ High school & $312(14)$ & $25(13)$ & $8(11)$ & 0.77 \\
\hline HS graduate & $746(35)$ & $73(37)$ & $29(39)$ & \\
\hline College+ & $1,095(51)$ & $97(50)$ & $37(49)$ & \\
\hline Rural location & $171(8)$ & $143(72)$ & $48(62)$ & $<0.0001$ \\
\hline Region & & & & $<0.0001$ \\
\hline Northeast & $802(37)$ & $39(20)$ & $5(6)$ & \\
\hline Midwest & $544(25)$ & $76(38)$ & $16(21)$ & \\
\hline South & $482(22)$ & $33(17)$ & $15(19)$ & \\
\hline West & $362(17)$ & $51(26)$ & $41(53)$ & \\
\hline MMSE score & $96(91-99)$ & $96(93-99)$ & $96(91-98)$ & 0.51 \\
\hline MMSE $<89$ & $382(17)$ & $20(10)$ & $11(14)$ & 0.02 \\
\hline Rankin grade $3+$ & $218(10)$ & $21(11)$ & $6(8)$ & 0.79 \\
\hline Leg edema $1+$ & $302(14)$ & $29(15)$ & $7(9)$ & 0.46 \\
\hline Current smoker & $354(16)$ & $24(12)$ & $19(25)$ & 0.04 \\
\hline No aerobic exercise & $866(40)$ & $91(46)$ & $31(40)$ & 0.23 \\
\hline Risk factors not at goal (any) & $1,209(56)$ & $116(59)$ & $36(48)$ & 0.25 \\
\hline $\mathrm{BP} \geq 140 / 90$ & $779(36)$ & $82(41)$ & $16(21)$ & 0.008 \\
\hline $\mathrm{LDL}>2.59 \mathrm{mmol} / \mathrm{L}$ & $689(32)$ & $69(35)$ & $23(30)$ & 0.56 \\
\hline Not on antithrombotic & $28(1)$ & $2(1)$ & $2(3)$ & 0.56 \\
\hline Pioglitazone group & $1,097(50)$ & $99(50)$ & $38(49)$ & 0.99 \\
\hline Baseline visit at home & $597(27)$ & $14(7)$ & $9(12)$ & $<0.0001$ \\
\hline
\end{tabular}

high-quality data and be effective participants in clinical stroke research, but that special procedures may be required for in-person visits. Solutions may include home visits [13] to relieve participants of the burden and risk of travel. Another is to front-load visits to the enrolment site to accommodate the fact that travel may become more difficult with time. A third is chauffeured transportation (and trying to tie research visits with other medical care). A fourth would be through the use of telemedicine.
Interestingly, we found that other measures of adherence were not adversely affected by the driving distance. Participants who lived at greater distances from the research site were less likely to become lost to follow-up. This was mainly attributable to lower rates of withdrawing consent among more distant participants, perhaps reflecting greater initial commitment to the study. Among patients who completed the in-person visit and had risk factors measured, we found no adverse effect of distance from residence to enrolment site in terms of control of 
Table 2. Participant retention and adherence to protocol by driving distance as a categorical variable

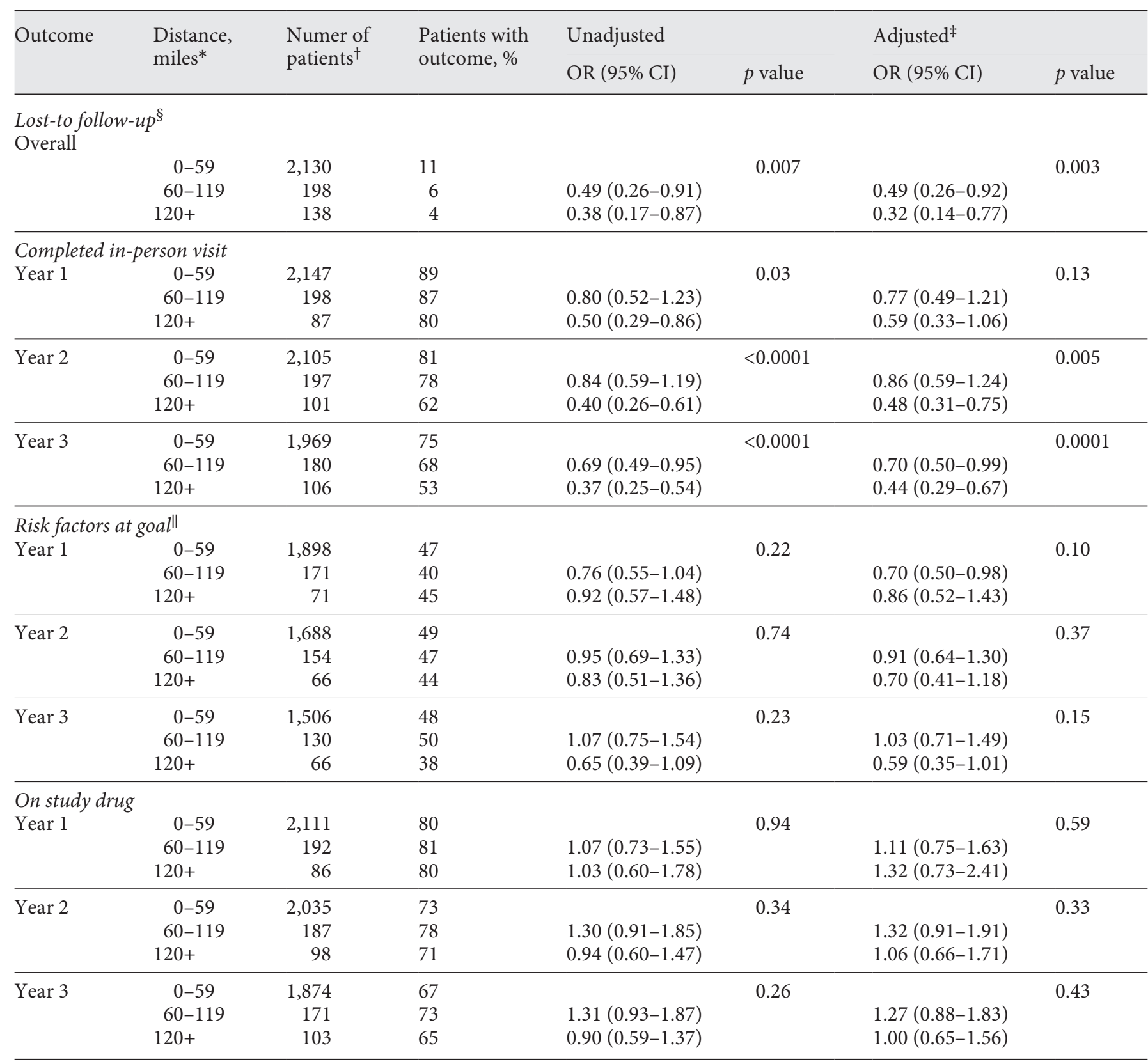

* Distance from participants' home to site using latest home address for lost-to-follow-up, or address at annual visit.

${ }^{\dagger}$ Number of participants (patients): loss-to-follow-up = all patients; annual visit = patients not deceased or exited at time of annual contact; risk factors at goal: patients with blood pressure, medication history and LDL measured at annual visit; on study drug: patients not removed from drug by Internal Safety Committee or deceased or exited at time of annual.

‡ Adjusted for baseline age, sex, lived alone, race, region, $3 \mathrm{MS}$ score $<89$, smoker, BP $>140 / 90$, baseline home visit.

$\S$ Consent withdrawn or missed at least 13 months of follow-up.

\| $\mathrm{BP} \leq 140 / 90 \mathrm{~mm} \mathrm{Hg}$, taking antithrombotic medication, and $\mathrm{LDL}<2.59 \mathrm{mmol} / \mathrm{L}$. 
Table 3. Effect of driving distance* as a continuous variable on participant retention and adherence to protocol

\begin{tabular}{|c|c|c|c|c|c|c|}
\hline \multirow[t]{2}{*}{ Outcome } & \multicolumn{3}{|c|}{ Unadjusted } & \multicolumn{3}{|c|}{ Adjusted } \\
\hline & $\mathrm{OR}^{\dagger}$ & $95 \% \mathrm{CI}$ & $p$ value & $\mathrm{OR}^{\ddagger}$ & $95 \% \mathrm{CI}$ & $p$ value \\
\hline Lost to follow-up & 0.96 & $0.92-1.00$ & 0.08 & 0.96 & $0.92-1.00$ & 0.06 \\
\hline \multicolumn{7}{|l|}{ Completed visit } \\
\hline Year 1 & 0.99 & $0.96-1.01$ & 0.20 & 0.99 & $0.96-1.01$ & 0.26 \\
\hline Year 2 & 0.97 & $0.96-0.99$ & 0.002 & 0.97 & $0.95-0.99$ & 0.004 \\
\hline Year 3 & 0.96 & $0.94-0.97$ & $<0.0001$ & 0.95 & $0.94-0.97$ & $<0.0001$ \\
\hline \multicolumn{7}{|l|}{ Risk factors at goal } \\
\hline Year 1 & 1.01 & $0.99-1.03$ & 0.50 & 1.00 & $0.97-1.02$ & 0.92 \\
\hline Year 2 & 1.01 & $0.99-1.03$ & 0.40 & 1.00 & $0.98-1.03$ & 0.93 \\
\hline Year 3 & 1.00 & $0.98-1.03$ & 0.95 & 0.99 & $0.97-1.02$ & 0.53 \\
\hline \multicolumn{7}{|l|}{ On study drug } \\
\hline Year 1 & 1.00 & $0.98-1.03$ & 0.83 & 1.01 & $0.98-1.04$ & 0.59 \\
\hline Year 2 & 0.99 & $0.98-1.01$ & 0.84 & 1.00 & $0.98-1.02$ & 0.91 \\
\hline Year 3 & 1.00 & $0.98-1.01$ & 0.69 & 1.00 & $0.98-1.01$ & 0.81 \\
\hline
\end{tabular}

* Distance from participant home to site defined as latest address for lost-to-follow-up outcome; address at the time of annual visit was due for annual outcomes.

${ }^{\dagger}$ OR for outcome per 30 miles of driving distance.

‡ OR for outcome per 30 miles of driving distance, adjusted for predictive features in Table 1.

blood pressure or LDL cholesterol and antithrombotic use. Similarly, we found that there was no effect of travel distance on study drug status over the first 3 years of participation. The IRIS protocol called for study drug resupplies to be mailed to participants, rather than dispensed at clinic visits, and this design may have enabled distant participants to remain on drug without the need for protracted travel to the site.

Our finding that in-person visits declined over time highlights the overall importance of engaging research participants and maximizing research convenience. Engaged, connected, and enthusiastic participants will bear more inconvenience than participants who are left with little sense that they are valued or respected. Convenient protocols that are easy to follow can capitalize on engagement and further protect against the dual curse of withdrawal of consent and nonadherence to treatment. In the IRIS trial, we engaged patients through frequent telephone contact, annual personal letters summarizing their progress in reducing their stroke risk, and newsletters to announce trial progress to all participants [14]. We minimized burden by requiring only 1 in-person visit annually [14].

Our research has some limitations. Our study cohort was comprised of patients who consented to participate in a clinical trial and this may reflect a selection bias that would tend to improve adherence. In particular, patients who consent to take part in secondary prevention research studies but live at a distance from the study center may be particularly capable of overcoming barriers to protocol adherence compared with patients who decline consent. As such, it is possible that our data underestimated the effect of distance on general stroke care, study drug adherence, and participation in in-person visits. Second, IRIS researchers were encouraged to visit homes and this may have resulted in an underestimation of the effect of distance on adherence with in-person visits [15]. The strength of this study is the large sample size of carefully collected data from participants distributed in all the geographical areas of the country, which allows generalization to trials conducted in the United States.

These results suggest that stroke researchers can enroll patients from rural communities without undue risk to the quality of their data. Protocol accommodations, including frequent telephone contact, home visits, and transportation assistance may be needed to safeguard rates of completion for in-person visits [16], but these accommodations require no sophisticated technology and are easy to implement.

\section{Funding Sources}

This study was supported by NIH grant U01NS044876. 


\section{References}

-1 Shiovitz TM, Bain EE, McCann DJ, Skolnick $\mathrm{P}$, Laughren T, Hanina A, et al: Mitigating the effects of nonadherence in clinical trials. J Clin Pharmacol 2016;56:1151-1164.

-2 Gencheva E, Sloan M, Leurgans S, Raman R, Harris Y, Gorelick P; AAASPS Investigators: Attrition and non-compliance in secondary stroke prevention trials. Neuroepidemiology 2004;23:61-66.

>3 Loftin WA, Barnett SK, Bunn PS, Sullivan P: Recruitment and retention of rural African Americans in diabetes research. Diabetes Educ 2005;31:251-259.

4 Leira EC, Phipps MS, Jasne AS, Kleindorfer DO: Time to treat stroke patients in rural locations as an underserved minority. Neurology 2017;88:422-423.

$>5$ Busija L, Tao LW, Liew D, Weir L, Yan B, Silver $G$, et al: Do patients who take part in stroke research differ from non-participants? Implications for generalizability of results. Cerebrovasc Dis 2013;35:483-491.

$\checkmark 6$ Leira EC, Lamb DL, Nugent AS, Ahmed A, Grimsman KJ, Clarke WR, et al: Feasibility of acute clinical trials during aerial interhospital transfer. Stroke 2006;37:2504-2507.
7 Saver JL, Starkman S, Eckstein M, Stratton SJ, Pratt FD, Hamilton S, et al: Prehospital use of magnesium sulfate as neuroprotection in acute stroke. N Engl J Med 2015;372:528-536.

$>8$ Kernan WN, Viscoli CM, Furie KL, Young LH, Inzucchi SE, Gorman M, et al: Pioglitazone after ischemic stroke or transient ischemic attack. N Engl J Med 2016;374:13211331.

$\checkmark 9$ Viscoli CM, Brass LM, Carolei A, Conwit R, Ford GA, Furie KL, et al: Pioglitazone for secondary prevention after ischemic stroke and transient ischemic attack: rationale and design of the insulin resistance intervention after stroke trial. Am heart J 2014;168:823-829. e6.

10 Polinski JM, Brookhart MA, Ayanian JZ, Katz JN, Kim SC, Lii J, et al: Relationships between driving distance, rheumatoid arthritis diagnosis, and disease-modifying antirheumatic drug receipt. Arthritis Care Res (Hoboken) 2014;66:1634-1643.

11 Lamont EB, Hayreh D, Pickett KE, Dignam JJ, List MA, Stenson KM, et al: Is patient travel distance associated with survival on phase ii clinical trials in oncology? J Natl Cancer Inst 2003;95:1370-1375.
2 Probst JC, Laditka SB, Wang JY, Johnson AO: Effects of residence and race on burden of travel for care: cross sectional analysis of the 2001 us national household travel survey. BMC Health Serv Res 2007;7:40.

13 Kernan W, Viscoli C, Brass L, Amatangelo M, Birch A, Clark W, et al: Boosting enrolment in clinical trials: validation of a regional network model. Clin Trials 2011;8:645-653.

14 Berge E, Stapf C, Al-Shahi Salman R, Ford GA, Sandercock P, van der Worp HB, et al: Methods to improve patient recruitment and retention in stroke trials. Int J Stroke 2016;11: 663-676.

15 Thayabaranathan T, Cadilhac DA, Srikanth VK, Fitzgerald SM, Evans RG, Kim J, et al: Maximizing patient recruitment and retention in a secondary stroke prevention clinical trial: lessons learned from the stand firm study. J Stroke Cerebrovasc Dis 2016;25: 1371-1380.

16 Pendlebury ST, Chen PJ, Welch SJ, Cuthbertson FC, Wharton RM, Mehta Z, et al: Methodological factors in determining risk of dementia after transient ischemic attack and stroke: (II) effect of attrition on follow-up. Stroke 2015;46:1494-1500. 